\title{
Efficacy of trichloroacetic acid in the treatment of anal intraepithelial neoplasia in HIV-positive and HIV-negative men who have sex with men
}

\author{
Jasmeet Chadha Singh, M.D. ${ }^{1}$, Victoria Kuohung, M.D. ${ }^{2}$, and Joel M Palefsky, M.D. ${ }^{3}$ \\ ${ }^{1}$ Department of Internal Medicine, St Luke's Roosevelt Hospital Center \\ ${ }^{2}$ Yale School of Medicine \\ ${ }^{3}$ Department of Medicine, University of California San Francisco
}

\begin{abstract}
BACKGROUND—Anal intraepithelial neoplasia (AIN), particularly AIN 3 is a precursor to anal cancer. Most cases of AIN are intra-anal, but few treatments for intra-anal AIN are currently available. Topical $85 \%$ trichloroacetic acid (TCA) is an inexpensive method used to treat peri-anal condyloma, a form of AIN 1, but its efficacy to treat intra-anal AIN as first-line therapy is unknown.
\end{abstract}

METHODS—Retrospective review of medical records was performed for all patients with AIN treated at the University of California San Francisco (UCSF) Anal Neoplasia Clinic with TCA as the first-line therapy from January 2000 to December 2004. Clearance was defined as the absence of AIN confirmed by high resolution anoscopy and cytology after up to four TCA treatments.

RESULTS-Thirty-five HIV-positive and 19 HIV-negative men met the enrollment criteria. In multivariate analysis, greater clearance was seen in patients $41-48$ years of age versus $>49$ years (OR: 8.4, CI: 1.1-94, p-value: 0.04). Among HIV-positive men, those with two or fewer lesions showed greater clearance (OR: 14.3, CI: 1.5-662, p-value: 0.01). 32\% of patients with AIN 2/3 cleared to no lesions. On a per-lesion basis, 73\% of AIN 1 and $71 \%$ AIN 2/3 cleared to no lesion or AIN 1 or less, respectively.

CONCLUSION-Topical 85\% TCA was safe and well tolerated. It was more effective in younger patients and among HIV-positive patients, those with 2 or fewer lesions. A high proportion of AIN 2/3 lesions responded to TCA treatment.

\section{Keywords}

Trichloroacetic acid; anal intraepithelial neoplasia; HIV, anal cancer, therapy

\section{Introduction}

Anal intraepithelial neoplasia (AIN) is a precancerous condition of anal canal, which like its counterpart in the cervix, cervical intraepithelial neoplasia (CIN), can progress to invasive cancer. Like CIN, there is a spectrum of AIN, graded as AIN I, II, or III depending on the extent of nuclear abnormalities. AIN I has nuclear abnormalities confined to the lower one-third of

Name and address for correspondence: Joel M. Palefsky, M.D, Professor of Medicine Box 0126 University of California, San Francisco San Francisco, CA- 94143 Phone number: 415-476-1574 joel.palefsky@ucsf.edu.

Address for reprints: Joel M. Palefsky, M.D. Professor of Medicine Box 0126 University of California, San Francisco San Francisco,

CA- 94143 
the epithelium. A common form of AIN 1 is condyloma acuminatum. Nuclear abnormalities in AIN 2 are confined to the lower two-thirds and in AIN 3 to the entire thickness of the epithelium.1, 2 AIN is strongly associated with human papillomavirus (HPV) infection. Both non-oncogenic (low-risk) types of HPV such as HPV 6 and HPV 11 and oncogenic (high-risk) types such as HPV 16 and HPV 18 are implicated in AIN 1 while AIN 3 and anal squamous cell cancer are associated with oncogenic HPV types.1 Beside HPV, other significant risk factors for anal cancer include smoking, anal fissure, anal fistula and hemorrhoids (greater than 12 episodes). 3 Most cases of AIN occur within the anal canal, particularly the anal transition zone, but AIN and anal cancer can also occur on the perianal skin.

The incidence of anal cancer has been increasing over the past four decades. ${ }^{4}, 5$ Though the incidence of anal cancer is low in the general population, ${ }^{6,7} 7$ it is reported to be as high as 35 per 100,000 in men who have a history of receptive anal intercourse. 8 Even higher risk for anal cancer has been observed in both men and women with HIV infection. $9^{-} 11$ Investigators estimate that the incidence of anal cancer among HIV-positive men who have sex with men (MSM) may be as high as 70-100 cases per 100,000 individuals.12 Like anal cancer, the incidence of AIN is also higher in HIV-positive men and women compared with the general population (relative risk [RR] of 60 and RR of 7.8, respectively).1, 13, 14 Most studies show that the introduction of highly active antiretroviral therapy (HAART) has not had an appreciable positive effect on prevalence or incidence of AIN in HIV-positive patients despite an increase in CD4 cell counts observed with therapy.2, $15^{-} 19$ Consistent with these findings, the incidence of anal cancer has continued to increase since the introduction of HAART.2, 15-19 Taken together, these data indicate that AIN and anal cancer will continue to remain a serious clinical problem, particularly among HIV-positive men and women.

Topical $85 \%$ TCA is one of the accepted cytotoxic therapies for condyloma and is recommended by Center for Disease Control (CDC) as one of the first-line treatments for condyloma acuminatum. ${ }^{20}$ The UCSF Anal Neoplasia Clinic has been using TCA the treatment of AIN for the past nine years. However, little is known about its efficacy and long-term clearance rates when used to treat intra-anal AIN of different grades of severity. TCA represents a potentially attractive treatment approach since it is inexpensive, is generally well tolerated by patients, has no systemic side-effects and is safe to use in pregnancy.6

The purpose of this study was to perform a retrospective chart review of patients seen in the UCSF Anal Neoplasia Clinic who received first-line 85\% TCA therapy for AIN of different grades in a population mostly consisting of HIV-positive MSM.

\section{Methods}

Retrospective review of medical records was performed for all patients at the UCSF Anal Neoplasia Clinic whose first-line treatment for AIN was TCA between January 1, 2000 and December 31, 2004. The inclusion criteria were age greater than 18 years; no treatment with TCA prior to January 1, 2000, lesions less than one square centimeter in size at the base, and patients' ability to return for regular follow-up visits. HIV-positive and HIV-negative patients were studied. Both external and internal lesions were treated. Both flat and raised lesions were treated if they met the inclusion criteria.

Patients were generally referred to the clinic after anal cytology smears performed by outside community physicians were reported to be abnormal. At the first visit, a detailed history and physical exam was performed on every patient. HIV-positive patients were questioned about their most recent CD4+ counts, HIV viral loads and their HIV history. A swab was inserted to collect cells for Thinprep ${ }^{\mathrm{TM}}$ anal cytology. After a digital rectal examination was performed, high resolution anoscopy (HRA) was performed as described previously using 3\% acetic acid 
and colposcopic magnification. ${ }^{12}$ The number and extent of lesions was recorded and a biopsy was performed of visible lesions. Anal cytology was used to determine the grade of AIN in patients in whom biopsy results were unsatisfactory or in whom biopsy could not be performed. When both an anal cytology and biopsy were available, the most severe grade of these two tests was used for analysis.

Most patients for whom TCA treatment was selected were seen within one to two months of the first visit to initiate their treatment. At the treatment visit, an anoscope was inserted and HRA was performed as described previously and the lesions were treated with $85 \%$ TCA by dipping the wooden stick end of a cotton swab into a cup containing about 250 microliters of TCA. The stick end was saturated with TCA and was inserted through the anoscope and directed to the lesion under colposcopic guidance. TCA was applied to the lesion repeatedly until the lesion turned dense white color.. The patient was counseled to expect anal bleeding for up to 2 weeks with bowel movements and to avoid straining or anal intercourse during this time.

During this time, each TCA application was followed by another appointment one to two months later, when the clinician reevaluated the patient to determine whether more TCA was necessary. First-line treatment consisted of up to four applications of TCA at one to two month intervals. Clearance was defined as the absence of AIN confirmed by absence of visible disease on HRA after up to four TCA treatments. If AIN cleared within four TCA applications, firstline therapy was considered successful and patients were then followed at three, six, and 12 months after the last evaluation at which they were found to be clear. If they still had AIN despite four treatments of TCA, they were considered to have failed the first-line treatment i.e. even if TCA was able to clear some lesions, it was not considered to be successful unless it cleared all AIN. Recurrence was defined as detection of AIN during the follow-up period after its resolution with the first-line therapy. Most patients who failed TCA therapy were then referred to receive infrared coagulation or surgery. Patients who cleared anytime during the first set of four TCA applications, but whose AIN recurred during three-, six-, or 12-month follow-ups, were also referred for infrared coagulation or surgery. Amongst the patients with AIN 2/3, we also measured the rate of regression of their AIN 2/3 to low-grade AIN (AIN 1). Hence, we defined cure of AIN 2/3 as conversion to AIN 1 as well as its complete disappearance. In addition to per-patient clearance, we measured clearance of AIN on a perlesion basis.

Statistical analysis was performed using SAS Version 9.1 (SAS Institute, Cary NC). SAS Procedure FREQ was used to compute the exact confidence intervals for the clearance and recurrence rates. SAS Procedure LOGISTIC was used to perform exact logistic regression.

\section{Results}

Fifty-four male patients met the inclusion criteria. The demographics of the study population are shown in Table 1. On the first visit, we determined the grade of AIN using either biopsy or cytology in forty seven out of fifty four patients. The remaining seven patients did have biopsy or cytology at the first visit during the follow-up course and were found to have AIN. Overall, 35 of 54 (65\%) patients were HIV-positive and 19 (35\%) were HIV-negative. 63\% of all HIV-positive patients were on HAART. There were no statistically significant differences between the HIV-positive and HIV-negative patients with respect to age, race, and number of lesions or severity of lesions (Table 1).

We examined complete clearance of lesions and clearance of AIN 2/3 lesions to AIN 1 or less on a per-patient and perlesion basis and the results are shown in Table 2 . Of 28 patients with histologically-confirmed AIN 2/3 lesions, 9 (32\%) resolved completely and $8(29 \%)$ resolved 
to AIN 1. Of 19 patients with histologically-confirmed AIN 1 lesions at the first visit, 6 (32\%) resolved completely. Of 55 histologically-confirmed AIN 2/3 lesions, 35 (64\%) resolved completely and $4(7 \%)$ resolved to AIN 1 . Of 70 histologically-confirmed AIN 1 lesions at the first visit, 51 (73\%) resolved completely. The odds of clearance for AIN 2/3 compared with AIN-1 on a per-lesion basis when adjusted for HIV status was 0.6 (95\% C.I 0.3-1.3, p= 0.2). The odds of clearance of lesions in HIV-positive individuals compared with HIV negative individuals on a per-lesion basis was 0.55 (95\% C.I. $-0.2-1.5, \mathrm{p}=0.2$ ).

Of the HIV-positive patients $34 \%$ achieved complete clearance with up to four treatments with TCA, compared with $47 \%$ of HIV-negative patients (OR $0.6,95 \%$ CI $0.2-2.1, \mathrm{p}=0.5$ ). Of the twenty-one patients who cleared AIN, nine patients required only two TCA treatments to clear completely, three patients required one treatment; four patients required three treatments and five patients cleared after four treatments. Patients who cleared required a mean of 2.5 treatments and median of 2 treatments.

Only 3 out of 54 (5\%) patients reported side effects with TCA, usually local discomfort and pain. One patient reported exacerbation of pre-existing psoriasis and none had serious side effects such as local ulceration, abscess or bleeding.

Risk factors associated with clearance of lesions on a per-patient basis after TCA therapy are shown in Table 3. In the analysis adjusted for HIV status, with all patients combined, men in the forty-one to forty-eight (41-48) years age group were more likely to achieve complete clearance with TCA (OR: 8.4, 95\% CI: 1.1-94, $\mathrm{p}=0.04$ ). The OR for clearance was also higher among men younger than 41 years compared with greater than 49 but the result did not reach statistical significance.

Among HIV-positive patients with one lesion, 48\% showed clearance compared with $18 \%$ of HIV-positive patients with 4 or more lesions. In univariate analysis on a per-patient basis, HIVpositive subjects showed significantly greater likelihood of clearance in patients with $\leq 2$ lesions (OR: 14.3, 95\%CI: 1.5-661.7, p-value: 0.01) compared with those with three to four lesions. Additionally, although not statistically significant, HIV-positive patients showing clearance of the lesions were found to have higher CD4+ counts when compared with those not showing clearance. A non-significant trend towards greater clearance was observed in the combined patient population with less than or equal to two versus three to four lesions (OR 3.5, 95\% CI: 0.90-14.7, p-value: 0.08).

Recurrence was defined as detection of AIN during the follow-up period after its resolution with the first line therapy and was observed within a mean period of 6 months in 15 (72\%) of 21 patients who had shown clearance previously. 6 of 9 (67\%) HIV-negative and 9 out of 12 (75\%) HIV-positive patients showed recurrence. In univariate analysis, the recurrence rate in patients with AIN 2/3 was higher than that in patients with AIN 1 (OR: 5.1, 95\% CI: 0.2-313.8, p-value: 0.6) but this was not statistically significant. A trend towards greater recurrence was also seen in younger patients compared with older age groups (OR: 3.2, 95\% CI 0.3-45.3, pvalue: 0.5 ). Recurrence was seen in ten out of sixteen patients with two or fewer lesion while it was seen in five out of six patients with three or more lesions (OR: 0.6, 95\% CI: 0.04-10, p-value: 1) but this was not statistically significant.

\section{Discussion}

AIN is a known precursor of invasive cancer ${ }^{21}$ with an estimated progression rate of AIN to squamous cell cancer of about 11 percent over a period of 42 months of follow-up. Although AIN 1 has a low risk of progression to malignancy, it has been observed that around $62 \%$ of HIV-positive and $36 \%$ of HIV-negative men with baseline AIN 1 progress to AIN 2-3 over a 
2-year follow-up period. ${ }^{22}$ Thus, there are potential benefits to treating both AIN 1 and AIN $2-3$.

Analogous to cervical cytology screening to identify and treat cervical intraepithelial neoplasia lesions before they progress to cervical cancer, anal cytology screening programs have been proposed to identify and treat AIN before it progresses to anal cancer in high-risk groups. ${ }^{23}$, ${ }^{24}$ Anal cytology is 69\% sensitive and 59\% specific in detecting any grade of anal dysplasia in HIV-positive men, while its sensitivity and specificity in HIV-negative men is $47 \%$ and $92 \%$ respectively when ASCUS is included as an abnormal cytology. ${ }^{25}$ Goldie et al have concluded that anal cytological screening in HIV-negative homosexual and bisexual men every 2-3 years and in HIV-positive men yearly, followed by treatment of high-grade AIN, would be optimal and cost-effective to prevent anal cancer. ${ }^{23,24}$

One of the barriers toward implementation of such a screening program has been the paucity of data on the treatment of AIN. Few studies have been published on the success of different treatment modalities to clear AIN lesions. Although it has not yet been proven in clinical trials, based on the analogy with the cervix, it is assumed that clearance of AIN will prevent progression of that lesion to anal cancer.

TCA is one of the accepted cytotoxic therapies for AIN, primarily AIN 1 in the form of condyloma acuminatum especially for smaller lesions. ${ }^{1,2}$ Few studies have compared TCA with other modalities. One study comprising 130 presumably HIV-negative men with penile condyloma randomly allocated to treatment with either TCA or cryotherapy concluded that there was no significant difference in the cure rates and subsequent recurrence rates between the two modalities. The clearance rates after five treatments of TCA and cryotherapy were $81 \%$ and $88 \%$ respectively and the recurrence rates over a period of 2 months with TCA and cryotherapy were $36 \%$ and $39 \%$ respectively. ${ }^{25}$

In our study, the clearance rates of AIN on a per-patient basis with TCA in HIV-negative and HIV-positive patients were 47 and 34 percent, respectively. On a per-lesion basis (AIN 1, AIN $2 / 3$ as well as lesions of unknown grade) the average rate of clearance of all lesions was somewhat higher at 64 percent. Defined as complete clearance as well as conversion to AIN 1 , the overall response rate of AIN 2/3 was also quite high at $71 \%$. While doing per-patient analysis, we did not biopsy every lesion after treatment but used very rigorous definitions for treatment response, i.e., complete absence of all lesions (and not merely reduction to a lower grade) after visualization with high resolution anoscopy. Our per-lesion clearance results are comparable to the clearance rates in the above mentioned earlier studies that determine the efficacy of TCA in treating genital warts. ${ }^{25}$ However, the studies are not strictly comparable since in the earlier study TCA was used to treat genital warts which are mainly caused by nononcogenic types of HPV ( 6 and 11) while AIN 2/3 is caused by oncogenic HPV types (16, 18, 31 and others).

We found TCA to be a safe outpatient treatment option for AIN as only $5 \%$ of treated patients complained of mild side-effects, i.e., local discomfort and flare-up of pre-existing psoriasis. Health care providers also find it safe and easy to use. Though there is no consensus regarding treatment of flat AIN 1, our earlier published data show high progression to AIN 2-3 over two years. Hence, we treated AIN 1 when feasible. ${ }^{22}$ TCA can be applied to more than one lesion at a time, allowing treatment of multiple lesions in a single visit. TCA is also relatively inexpensive. Our results show that TCA may be a useful first-line therapy in the patients under 50 years of age with one to two lesions; clearance was substantially lower among those with more than 2 lesions. Although patients presenting with this limited degree of disease would be relatively rare in a referral population such as ours, we expect that these patients would be more common in a population that was being actively and routinely screened for AIN. 
Neither the grade nor the HIV status was significantly associated with response rates, although this may reflect the small sample size of the study. TCA is probably less effective in large bulky condylomas, which despite being classified as AIN 1, are hyperkeratotic and would respond poorly due to poor penetration at the base of the lesion.

Among those who did clear, the per-patient recurrence rate was quite high, with our data showing a trend to recurrence that was higher in patients with AIN 2-3. The recurrence rate was likely overestimated since any new lesion that was diagnosed after initial clearance was counted as recurrent, and many of these may have been new lesions unrelated to the treated lesions. Whether or not these lesions are truly recurrent, these data do point to the need to continue to monitor patients even after successful therapy.

To our knowledge, this is the first study that demonstrates the efficacy of TCA in long-term clearance of AIN. A number of other treatment options are available for AIN for whom TCA is not suitable or who fail TCA, including immunomodulation, locally ablative procedures and surgery. ${ }^{26}$ Earlier studies have shown that surgery for AIN is associated with a poor outcome in HIV-positive patients because of high persistence and recurrence rates after single procedure and significant post-operative morbidity. ${ }^{27-29}$ In another study, 23 out of 29 HIV-positive patients showed persistent/recurrent disease $(\mathrm{p}<0.003)$ after surgery. ${ }^{30}$ This result should be interpreted with caution since surgery was reserved for patients who had the largest lesions or who had failed other treatment modalities. However, a more recent retrospective study from the same group showed better results. ${ }^{31}$ After an average follow up for 44 months, $78 \%$ of patients treated surgically remained free of AIN 2-3 and the rate of progression to SCC was only $1.2 \%$. Treatment complications included anal stenosis, bleeding requiring reoperation, anal fissure, myocardial infarction and cellulitis.

Infrared coagulation (IRC) is also an effective treatment for AIN in both HIV-positive and HIV-negative men. ${ }^{32-34}$ It has been reported that the cure rate of AIN 2-3 with IRC with the average number of lesions being 1.6 in HIV-positive MSM was $72 \% .{ }^{33}$ After IRC, $65 \%$ of treated HIV-positive individuals developed new AIN 2-3 in a mean of 217 days and $28 \%$ persisted. After second and third treatments the new/persistent lesions dropped to 58\% and $40 \%$ respectively. In HIV-negative MSM, IRC produced even better results with clearance of $81 \%$ and recurrence rates of $53 \%$ after a mean of 238 days, $28 \%$ and $0 \%$ after first, second and third ablations. ${ }^{34}$ The clearance results of our per-lesion analysis were comparable to these clearance rates. Though IRC is safe and effective, it requires local anesthesia or sedation, may require several applications and is more expensive than TCA. Moreover, use of TCA allows people with almost no additional training to treat AIN identifiable on HRA, whereas other modalities such as IRC and surgery require further training and expertise. However, regular follow-up after treatment with TCA is paramount to ensure regression/clearance of previously treated lesions and to address any recurrent or newly appearing lesions. Hence TCA treatment may require more patient visits compared with IRC.

One of the main limiting factors of our study was the small sample size. Normal cytology and visual absence of all lesions were considered the criteria for response, but random biopsying may have yielded results of AIN in some patients, leading us to overestimate the per-lesion and per-patient response rates. Conversely, we may also have overestimated recurrence after treatment since lesions detected during follow-up could have been newly appearing lesions and not necessarily the recurrence of the treated lesion. Other limitations of the study include the generalizability of the results since we selected patients with limited disease and treatment at more frequent intervals may have led to better efficacy. Finally, in some patients, we used cytology to classify the grade of disease prior to TCA therapy. Anal cytology is likelier to underestimate the grade of true disease rather than overestimate it. Therefore, it is possible that 
we underestimated the proportion of patients with AIN 2/3, but since this was a small proportion of our patients, the effect on our data is likely to be limited.

This is the first study that describes the efficacy and safety of TCA in the treatment of AIN. Given its ease of use, low cost and good safety profile, TCA represents a reasonable first-line therapy with carefully selected patients. Larger, prospective randomized studies are needed to determine efficacy of TCA for treatment of AIN in comparison with other treatment modalities, and ultimately to reduce the risk of progression of AIN to invasive cancer.

\section{Conclusion}

TCA may be a safe, inexpensive and effective treatment in patients with two or fewer discrete AIN lesions, particularly for patients under 50 years of age. Follow-up is needed because of the high potential for lesion recurrence.

\section{Acknowledgments}

The authors would like to thank Barbara Grimes and Su-Chun Cheng for their valuable help in the data analysis.

Supported by American Cancer Society grant SPRSG-03-242-01 and NIH/NCRR UCSF-CTSA Grant UL1 RR024131.

\section{References}

1. Lacey CJ. Therapy for genital human papillomavirus-related disease. J Clin Virol Mar;2005 32(Suppl 1):S82-90. [PubMed: 15753016]

2. Hagensee ME, Cameron JE, Leigh JE, Clark RA. Human papillomavirus infection and disease in HIVinfected individuals. Am J Med Sci Jul;2004 328(1):57-63. [PubMed: 15254442]

3. Holly EA, Whittemore AS, Aston DA, Ahn DK, Nickoloff BJ, Kristiansen JJ. Anal cancer incidence: genital warts, anal fissure or fistula, hemorrhoids, and smoking. J Natl Cancer Inst Nov 15;1989 81 (22):1726-1731. [PubMed: 2810388]

4. Maggard MA, Beanes SR, Ko CY. Anal canal cancer: a population-based reappraisal. Dis Colon Rectum Nov;2003 46(11):1517-1523. discussion 1523-1514; author reply 1524. [PubMed: 14605572]

5. Johnson LG, Madeleine MM, Newcomer LM, Schwartz SM, Daling JR. Anal cancer incidence and survival: the surveillance, epidemiology, and end results experience, 1973-2000. Cancer Jul 15;2004 101(2):281-288. [PubMed: 15241824]

6. Melbye M, Frisch M. The role of human papillomaviruses in anogenital cancers. Semin Cancer Biol Aug;1998 8(4):307-313. [PubMed: 9870037]

7. Jemal A, Murray T, Samuels A, Ghafoor A, Ward E, Thun MJ. Cancer statistics, 2003. CA Cancer J Clin Jan-Feb;2003 53(1):5-26. [PubMed: 12568441]

8. Daling JR, Weiss NS, Hislop TG, et al. Sexual practices, sexually transmitted diseases, and the incidence of anal cancer. N Engl J Med Oct 15;1987 317(16):973-977. [PubMed: 2821396]

9. Uronis HE, Bendell JC. Anal cancer: an overview. Oncologist May;2007 12(5):524-534. [PubMed: 17522240]

10. Durante AJ, Williams AB, Da Costa M, Darragh TM, Khoshnood K, Palefsky JM. Incidence of anal cytological abnormalities in a cohort of human immunodeficiency virus-infected women. Cancer Epidemiol Biomarkers Prev Jul;2003 12(7):638-642. [PubMed: 12869403]

11. Williams AB, Darragh TM, Vranizan K, Ochia C, Moss AR, Palefsky JM. Anal and cervical human papillomavirus infection and risk of anal and cervical epithelial abnormalities in human immunodeficiency virus-infected women. Obstet Gynecol Feb;1994 83(2):205-211. [PubMed: 8290181]

12. Chin-Hong PV, Palefsky JM. Natural history and clinical management of anal human papillomavirus disease in men and women infected with human immunodeficiency virus. Clin Infect Dis Nov 1;2002 35(9):1127-1134. [PubMed: 12384848] 
13. Frisch M, Biggar RJ, Goedert JJ. Human papillomavirus-associated cancers in patients with human immunodeficiency virus infection and acquired immunodeficiency syndrome. J Natl Cancer Inst Sep 20;2000 92(18):1500-1510. [PubMed: 10995805]

14. Goedert JJ, Cote TR, Virgo P, et al. Spectrum of AIDS-associated malignant disorders. Lancet Jun 20;1998 351(9119):1833-1839. [PubMed: 9652666]

15. Abbasakoor F, Boulos PB. Anal intraepithelial neoplasia. Br J Surg Mar;2005 92(3):277-290. [PubMed: 15736144]

16. Wienecke R, Brockmeyer NH, Kreuter A. Human papilloma virus-induced disease in HIV-positive patients. Hautarzt Nov;2006 57(11):994-998. [PubMed: 17051407]

17. Horster S, Thoma-Greber E, Siebeck M, Bogner JR. Is anal carcinoma a HAART-related problem? Eur J Med Res Apr 30;2003 8(4):142-146. [PubMed: 12765859]

18. Palefsky JM, Holly EA, Efirdc JT, et al. Anal intraepithelial neoplasia in the highly active antiretroviral therapy era among HIV-positive men who have sex with men. Aids Sep 2;2005 19(13): 1407-1414. [PubMed: 16103772]

19. Piketty C, Darragh TM, Heard I, et al. High prevalence of anal squamous intraepithelial lesions in HIV-positive men despite the use of highly active antiretroviral therapy. Sex Transm Dis Feb;2004 31(2):96-99. [PubMed: 14743072]

20. Workowski KA, Berman SM. Sexually transmitted diseases treatment guidelines, 2006. MMWR Recomm Rep Aug 4;2006 55(RR-11):1-94. [PubMed: 16888612]

21. Watson AJ, Smith BB, Whitehead MR, Sykes PH, Frizelle FA. Malignant progression of anal intraepithelial neoplasia. ANZ J Surg Aug;2006 76(8):715-717. [PubMed: 16916390]

22. Palefsky JM, Holly EA, Hogeboom CJ, et al. Virologic, immunologic, and clinical parameters in the incidence and progression of anal squamous intraepithelial lesions in HIV-positive and HIV-negative homosexual men. J Acquir Immune Defic Syndr Hum Retrovirol Apr 1;1998 17(4):314-319. [PubMed: 9525431]

23. Palefsky JM. Anal squamous intraepithelial lesions in human immunodeficiency virus-positive men and women. Semin Oncol Aug;2000 27(4):471-479. [PubMed: 10950374]

24. Palefsky JM. Anal squamous intraepithelial lesions: relation to HIV and human papillomavirus infection. J Acquir Immune Defic Syndr Aug 1;1999 21(Suppl 1):S42-48. [PubMed: 10430218]

25. Palefsky JM, Holly EA, Hogeboom CJ, Berry JM, Jay N, Darragh TM. Anal cytology as a screening tool for anal squamous intraepithelial lesions. J Acquir Immune Defic Syndr Hum Retrovirol Apr 15;1997 14(5):415-422. [PubMed: 9170415] 


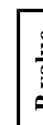

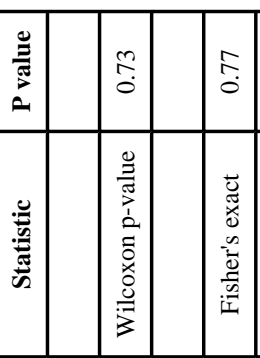
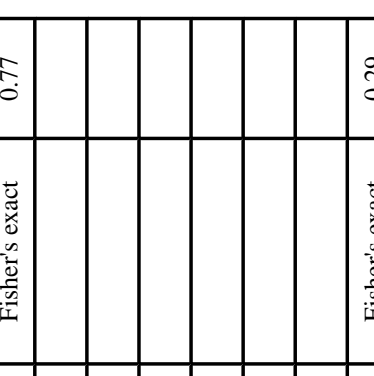

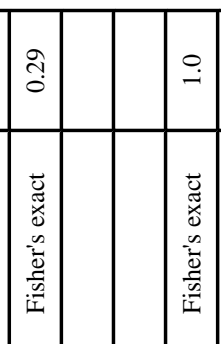
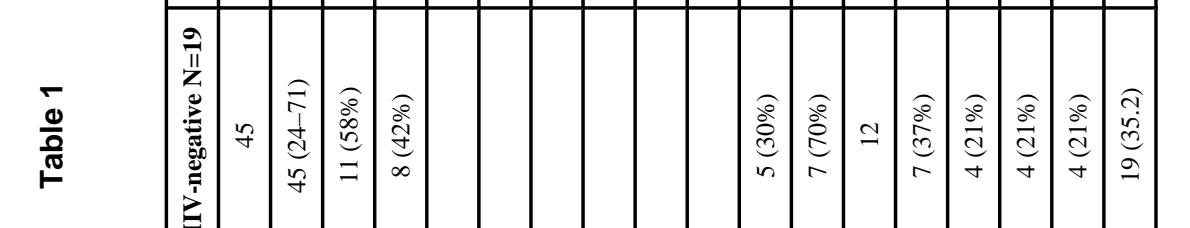

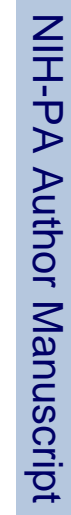

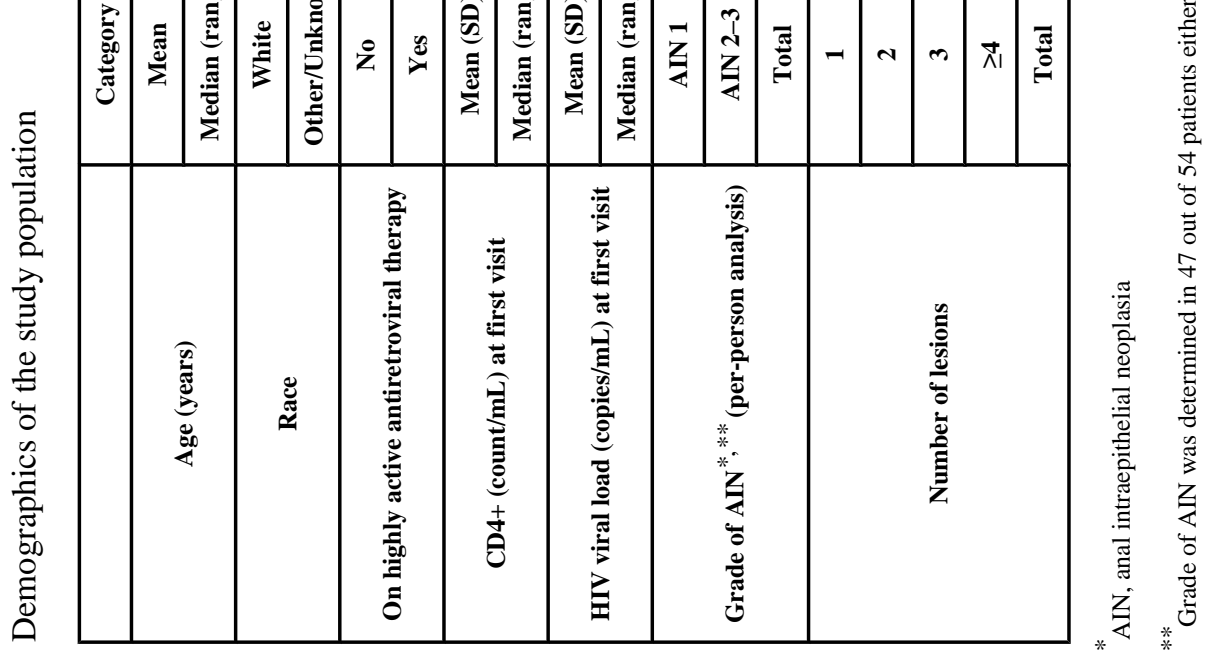

J Acquir Immune Defic Syndr. Author manuscript; available in PMC 2010 December 1. 
Table 2

Proportion of patients and lesions showing regression and clearance after treatment with $85 \%$ trichloroacetic acid

\begin{tabular}{|l|l|l|l|}
\hline Lesion grade at first visit & Complete clearance N (\%) & Regression to AIN 1 N (\%) & Regression to AIN 1 or less N (\%) \\
\hline Per-patient & & & \\
\hline AIN 1 & $6 / 19(32 \%)$ & - & - \\
\hline AIN 2/3 & $9 / 28(32 \%)$ & $8 / 28(29 \%)$ & $17 / 28(61 \%)$ \\
\hline Per-lesion & & & \\
\hline AIN 1 & $51 / 70(73 \%)$ & - & - \\
\hline AIN 2/3 & $35 / 55(64 \%)$ & $4 / 55(7 \%)$ & $39 / 55(71 \%)$ \\
\hline
\end{tabular}

AIN, Anal intraepithelial neoplasia 


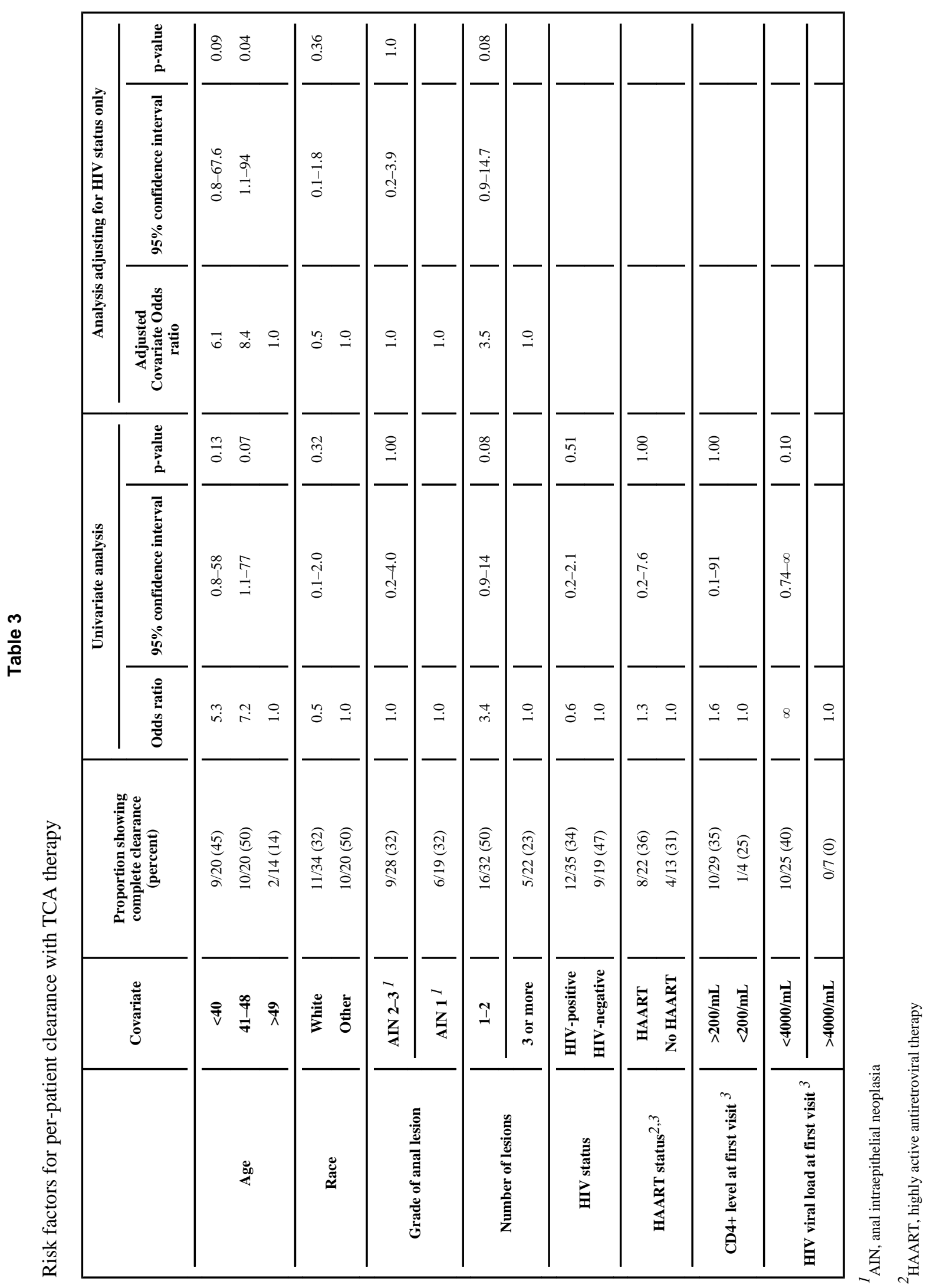

J Acquir Immune Defic Syndr. Author manuscript; available in PMC 2010 December 1. 
